\title{
Midlife Arterial Stiffness and Brain Activation During Working Memory Task
}

\author{
Kayla A. Steward ${ }^{1}$ Evan Pasha ${ }^{2}$, Katyoon Goudarzi ${ }^{1}$, Ahmed Elmenshawy ${ }^{2}$, Mohammed Alkatan ${ }^{2}$, \\ Astrid Villalpando ${ }^{1}$, Hirofumi Tanaka ${ }^{2}$ and Andreana P. Haley ${ }^{1,3, *}$
}

\author{
${ }^{I}$ Department of Psychology, ${ }^{2}$ Department of Kinesiology and Health Education, ${ }^{3}$ Imaging Research Center, University \\ of Texas at Austin, Austin, TX, USA
}

\begin{abstract}
Objective: Arterial stiffness is associated with cognitive decline and may serve as an early marker of brain vulnerability. In search of potential early intervention targets, the present study examined the neural correlates of working memory in relation to arterial stiffness in middle-aged, cognitively healthy adults. Methods: Twenty-eight adults, ages 4060 years, completed a 2-Back verbal working memory task during fMRI. Arterial stiffness was measured using the $\beta$ stiffness index via simultaneous ultrasound and applanation tonometry on the carotid artery. Mean task-related activation intensity was determined for 12 a priori regions of interest (ROI). Statistical analyses included partial correlations, controlling for hypertension status and antihypertensive medication. Results: Arterial stiffness was correlated negatively with task-related activation in 3 ROIs: left precentral gyrus/BA $6(\mathrm{r}=-0.64, \mathrm{p}<0.001)$, left precentral/middle frontal gyrus $(\mathrm{r}=-0.62, \mathrm{p}=0.001)$ and left superior parietal lobule/BA $7(\mathrm{r}=-0.48, \mathrm{p}=0.013)$. There was also a negative association between arterial stiffness and task-related activation in the right superior frontal gyrus/BA $6(\mathrm{r}=-0.45, \mathrm{p}=0.023)$. Conclusions: Greater arterial stiffness was significantly associated with decreased task-related brain activation during a verbal working memory task, possibly reflecting increased vulnerability for cognitive impairment. Arterial stiffness should be investigated further as an early marker of cognitive risk and a potential target for early intervention.
\end{abstract}

Keywords: Arterial stiffness, $\beta$-stiffness index, working memory, fMRI, BOLD, middle-aged adults, aging.

\section{INTRODUCTION}

Cognitive functioning is one of the most important determinants of functional ability, and quality of life [1]. Dementia currently affects approximately 24 million people worldwide and is estimated to reach 42 million by 2020 [2]. Accordingly, it is becoming increasingly important to understand the pathogenesis of dementia and identify early risk factors that can be targeted for primary prevention. Accumulating evidence points to vascular risk factors as key players in the development of dementia [3].

Arterial stiffness, which is characteristic of the aging process, is an independent risk factor for coronary heart disease, renal disease, stroke, and total mortality [4]. Arterial stiffening results from changes in both the structure and cellular elements of the vessel wall and subsequent deterioration of elastic fibers [5]. Recent studies have associated arterial stiffness with global cognitive decline [6-9], suggesting that arterial stiffness may be a target for early intervention for cognitive decline and dementia. Most previous studies to date have focused on elderly populations, [6-9] yet in order to develop effective early interventions it is critical to identify markers of cognitive vulnerability in younger samples, particularly in middle-aged adults. This poses a problem with traditional neuropsychological measures, which may lack the sensitivity to detect early, subtle changes in

*Address correspondence to this author at the Department of Psychology, The University of Texas at Austin, 108 East Dean Keeton, Stop A8000, Austin, TX 78712, USA; Tel: +1 512232 0863;

Fax: +1 512471 6175; Email: haley@austin.utexas.edu functioning in cognitively healthy individuals. Neuroimaging is a uniquely sensitive and robust measure that could potentially detect changes in cerebrovascular functioning before behavioral decline manifests [10].

In the present study, we determined the relationship between arterial stiffness and cerebrovascular response to cognition in an apparently and cognitively healthy, middle-aged sample. To address this aim, we utilized blood-oxygen-leveldependent functional magnetic resonance imaging (BOLD fMRI) to assess brain activation during a verbal working memory (VWM) task. Based on previous findings of diminished cerebral blood flow in task-related areas during working memory (WM) in a sample with vascular risk, [11] we hypothesized that increased arterial stiffness would relate to decreased BOLD response in task-related brain regions.

\section{METHODS}

\section{Participants}

Thirty-seven right-handed participants aged 40 to 60 years were recruited from Austin, TX with flyers and newspaper advertisements. In an effort to recruit participants with varying levels of arterial stiffness, we chose to target individuals with varying risk for cardiovascular disease. We used hypertension status as a convenient marker for cardiovascular risk [12], targeting participants who were hypertensive, normotensive with genetic risk of hypertension, and normotensive with no genetic risk. Consistent with previous studies, family history of hypertension, defined as hypertension before age 60 years in a first-degree relative, was used 
as a surrogate marker of genetic risk for hypertension [13]. Family history of hypertension was confirmed using the Ohio Blood Pressure History Survey, which has a sensitivity of $95.4 \%$, specificity of $92.4 \%$, and accuracy of $94.2 \%$ [14]. Hypertension was defined as a diagnosis from a physician, use of antihypertensive medication or meeting The Seventh Report of the Joint National Committee on Prevention, Detection, Evaluation, and Treatment of High Blood Pressure (JNC 7) hypertension guidelines: systolic pressure $\geq 140$ $\mathrm{mmHg}$ and/or diastolic pressure $\geq 90 \mathrm{mmHg}$ [15].

Participants were excluded from the study if they were smokers or had a history of neurological disease (i.e., large vessel stroke, seizure disorder, Parkinson's disease, clinically significant traumatic brain injury, multiple sclerosis, or brain infection/meningitis), major psychiatric illness (e.g. schizophrenia, bipolar disorder), substance abuse (i.e., diagnosed abuse and/or previous hospitalization for substance abuse), or MRI contraindications.

Of the thirty-seven participants recruited, two were unable to complete the imaging visit, one due to anxiety and another due to being overweight. Three more participants exhibited below chance performance on the fMRI task and were removed from the analyses. Arterial stiffness could not be assessed on four additional participants. The final sample included 28 participants.

The local Institutional Review Board approved the study, and all volunteers provided written informed consent before enrollment. Participants completed a medical history interview with one of the team members. Medical conditions and treatments were coded as either present or absent according to participants' self-report. Handedness was confirmed using the Edinburgh Handedness Questionnaire [16].

\section{Working Memory Paradigm}

Working memory was assessed using a verbal n-Back task [17], which consisted of a series of single consonants presented visually for $500 \mathrm{~ms}$ each and a $2500 \mathrm{~ms}$ interstimulus interval. Consonants were arranged in random order from a list of all consonants except "L" due to ambiguity in the lowercase form. Participants were asked to determine whether each stimulus was a target letter. Responses were collected using a two-button MR-compatible response box. Participants used their right index finger to indicate an affirmative response and their right middle finger to indicate a negative response. A 0 -Back control condition was alternated with a 2-Back condition in a block design. An imaging run consisted of three blocks of the 0-Back, three blocks of the 2-Back, and three blocks of rest presented in alternating order. The task lasted approximately 6 minutes. Each session included two runs of the task. The 0 -Back condition consisted of three blocks of 12 consonants of random case and order, $33 \%$ of which were targets. Targets were defined as an uppercase or lowercase letter "H." The 2-Back condition consisted of three blocks, each containing 15 consonants of random case and order, $33 \%$ of which were targets. A letter was a target if it was the same as the letter presented two stimuli earlier regardless of the case (e.g., s, G, $\underline{S}, d, V$, D ...). Task performance was assessed by measuring accuracy rates and mean reaction time for all correct trials. Dur- ing the rest condition participants rested for 30 seconds while focusing on a fixation cross in the middle of the screen.

\section{Procedures}

Brachial blood pressure was measured using an automatic blood pressure device (VP-2000, Colin Medical, San Antonio, TX) that uses the modified oscillometric pressure sensor method [18].

Arterial stiffness of the common carotid artery was assessed with the $\beta$-stiffness index [19]. Common carotid artery diameter was measured from the images derived from an ultrasound machine (iE 33, Philips, Bothell, WA) equipped with a high-resolution linear-array transducer. Simultaneously, contralateral carotid artery pressure was acquired via applanation tonometry (Millar TCB-500, Millar Instruments, Houston, TX) under quiet conditions with participants in the supine position. All ultrasound images were analyzed by the same investigator using automated edge-detection software (Vascular Research Tool Carotid Analyzer, Medical Imaging Applications, Coralville, IA) that allowed the investigator to find maximal systolic expansion and basal (minimum) diastolic diameters of the carotid artery. Carotid blood pressure signal obtained by applanation tonometer was calibrated by equating the carotid mean and diastolic blood pressure to the brachial artery value because the baseline levels of carotid blood pressure are subjected to hold-down force. $\beta$-stiffness index is calculated from the ratio of the natural logarithm of SBP/DBP to the relative change in arterial diameter.

All participants completed standard clinical neuropsychological instruments with established reliability and validity [20]. These measures assessed global cognitive functioning (Mini Mental Status Exam [21]; Wechsler Abbreviated Scale of Intelligence [22]) and emotional functioning (Beck Depression Inventory-II [23]; State Trait Anxiety Inventory [24]). Tests were administered and scored by a trained research assistant using standard administration and scoring criteria.

Each imaging session included at least six blocks of 0 Back/2-Back practice, two imaging runs of the 2-Back task, and $\mathrm{T}_{1}$-weighted imaging for anatomical reference. If necessary, more practice blocks were administered. E-Prime software (Psychology Software Tools, Inc., Pittsburgh, PA) was used to present the 2-Back task, which was back-projected onto a screen positioned at the participant's head and viewed through a double-mirror attached to the head coil. An MRcompatible response box collected participants' responses.

\section{MRI Data Acquisition}

MRI data for each participant were acquired in a single session on a 3 T GE Signa Excite MRI scanner equipped with a standard head coil. A whole brain echo-planer imaging (EPI) sequence (TR $=3000 \mathrm{~ms}, \mathrm{TE}=30 \mathrm{~ms}, \mathrm{FOV}=24 \mathrm{~cm}^{2}$, 64 x 64 matrix, 42 axial slices, $3 \mathrm{~mm}$ slice thickness, $0.3 \mathrm{~mm}$ gap) was used for functional imaging. Structural imaging included a high-resolution Spoiled Gradient Echo (SPGR) sequence $\left(256 \times 256\right.$ matrix, FOV $=24 \mathrm{~cm}^{2}, 1 \mathrm{~mm}$ slice thickness, 0 gap) anatomical scan of the entire brain in the sagittal plane. 


\section{Data Preprocessing}

All EPI images were processed using Analysis of NeuroImages (AFNI) software [25]. Each time series was spatially registered to the sixth volume of the session to reduce the effects of head movement. This AFNI 3-dimensional registration program also provides information on displacement and rotation for each volume that was used later to further correct motion. Data pre-processing also included adjustment for differences in adjacent slice timing due to interleaved slice acquisition, temporal smoothing, spatial filtering, and transformation to standard stereotaxic space [26]. Task-related brain activation was determined using voxelwise multiple regression analyses with the following parameters: a 0-Back/2-Back reference waveform convolved with a gamma function and covariates accounting for instruction screens, head movement, and linear trends.

A priori regions of interest (ROIs) were created from areas associated with a verbal 2-Back task in a healthy sample [17]. Coordinates from identified ROIs [17] were used to create spherical a priori ROIs, each with a conservative fivemillimeter radius used to minimize inclusion of white matter. The coordinates and their cortical areas are presented in (Table 2). The ROIs were applied to individual data to determine mean task-related activation intensity in each region.

\section{Statistical Analyses}

All variable distributions were examined using the Shapiro-Wilk test of normality recommended for small samples. Descriptive statistics were calculated for all demographic and clinical variables (Table 1).

The relationship between $\beta$-stiffness index and taskrelated brain activation was assessed for each ROI using partial correlations controlling for use of antihypertensive medication and hypertension status (normotension, normotension with family history of hypertension, or hypertension). Both hypertension [11] and genetic risk of hypertension [27] have been related to subclinical dysfunction in the neural systems supporting WM. A Sidak-corrected $\alpha$ level of 0.015 was used as the criterion of statistical significance to account for multiple comparisons and preserve the 5\% Type I error rate. Exploratory correlations $(\alpha=0.05)$ between activation and task accuracy and activation and reaction time were performed in the three areas in which activation significantly correlated with $\beta$-stiffness index. Data were analyzed using SPSS 16.0 computer software (SPSS Inc., Chicago, IL).

\section{RESULTS}

Descriptive statistics regarding the clinical and demographic variables are presented in (Table 1). $\beta$-stiffness index did not significantly correlate with age, body mass index, brachial blood pressure, IQ, global cognitive performance, memory, or emotional functioning (current level of depressive symptoms, state and trait anxiety). $\beta$-stiffness index was not different between men and women or between hypertension status groups.

Mean accuracy on the verbal 2-Back task was $80 \pm 10 \%$, and mean reaction time was $1124 \pm 304 \mathrm{~ms}$. $\beta$-stiffness index did not significantly correlate with task accuracy or reaction time. All participants moved less than $1.5 \mathrm{~mm}$ per imaging run.

Table 1. Sample Characteristics

\begin{tabular}{|c|c|}
\hline Characteristic & \# or Mean \\
\hline Males, $\%$ & $64.3 \%$ \\
\hline Age, year & $50 \pm 6$ \\
\hline Education, year & $16 \pm 3$ \\
\hline \multicolumn{2}{|l|}{ Race, \% } \\
\hline Caucasian, non-Hispanic & $64.3 \%$ \\
\hline Hispanic & $17.9 \%$ \\
\hline African American, non-Hispanic & $7.1 \%$ \\
\hline Asian American & $3.6 \%$ \\
\hline Other & $3.6 \%$ \\
\hline No response & $3.6 \%$ \\
\hline Beta-Stiffness Index, U & $5.9 \pm 1.6$ \\
\hline Body Mass Index, $\mathrm{kg} / \mathrm{m}^{2}$ & $27.3 \pm 5.0$ \\
\hline Brachial SBP, mmHg & $127 \pm 19$ \\
\hline Brachial DBP, mmHg & $77 \pm 10$ \\
\hline Brachial MAP, mmHg & $96 \pm 14$ \\
\hline Family History of Hypertension, $\%$ & $39 \%$ \\
\hline Hypertension, \% & $25 \%$ \\
\hline \multicolumn{2}{|l|}{ Antihypertensive Medication, $\%$} \\
\hline ACE Inhibitors & $21.4 \%$ \\
\hline Beta Blockers & $10.7 \%$ \\
\hline Calcium Blocker & $3.6 \%$ \\
\hline FSIQ & $113 \pm 13$ \\
\hline Mini Mental Status Exam & $28.3 \pm 1.5$ \\
\hline Beck Depression Inventory-II & $6.1 \pm 5.5$ \\
\hline State Trait Anxiety Inventory-State & $45.0 \pm 6.1$ \\
\hline State Trait Anxiety-Trait & $49.3 \pm 8.6$ \\
\hline
\end{tabular}

$\beta$-stiffness index was significantly correlated with taskrelated activation in three of the twelve ROIs: left precentral gyrus/BA $6(\mathrm{r}=-0.64, \mathrm{p}<0.001)$, left precentral/middle frontal gyrus $(\mathrm{r}=-0.62, \mathrm{p}=0.001)$, and left superior parietal lobule/BA 7 ( $\mathrm{r}=-0.48, \mathrm{p}=0.013)$ (Figs. 1-3). In addition, there was a strong trend between increased $\beta$-stiffness index and decreased task-related activation in the right superior frontal gyrus/BA $6(\mathrm{r}=-0.45, \mathrm{p}=0.023)$.

Exploratory analyses revealed significant correlations between accuracy on the 0 -Back task blocks and activation in the left precentral gyrus/BA $6(\mathrm{r}=0.49, \mathrm{p}=0.009)$ and in the left superior parietal lobule/BA $7(\mathrm{r}=0.44, \mathrm{p}=0.021)$, as well 
Table 2. Coordinates of a Priori Regions of Interest and their Cortical Areas

\begin{tabular}{|c|c|c|c|}
\hline Right Superior Frontal Gyrus, BA 6 & 13 & 8 & 54 \\
\hline Right Middle Frontal Gyrus, BA 9 & 37 & 37 & 33 \\
\hline Left Inferior Frontal Gyrus & -47 & 6 & 15 \\
\hline Left Insula & -32 & 20 & 8 \\
\hline Left Precentral Gyrus, BA 6 & -39 & -1 & 29 \\
\hline Left Precentral Gyrus, BA 6 & -47 & -2 & 43 \\
\hline Right Caudate Nucleus & 18 & 1 & 23 \\
\hline
\end{tabular}

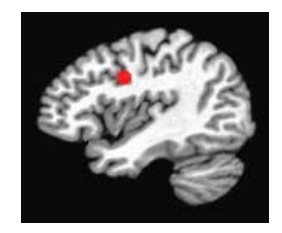

Left Precentral Gyrus/BA 6

$-39-129$

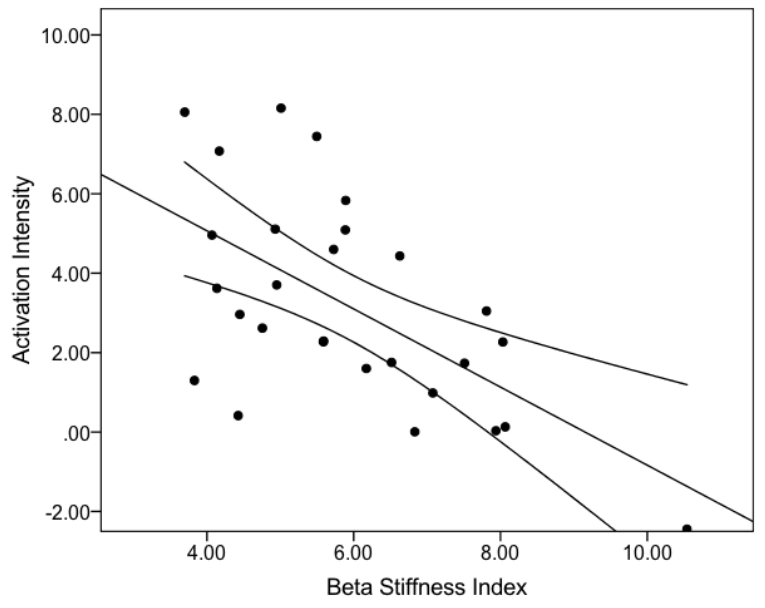

Fig. (1). Activation intensity versus BSI in the left precentral gyrus/BA 6.

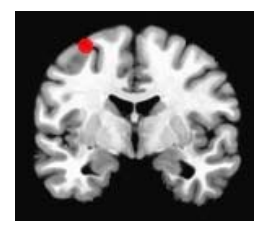

Left Precentral/ Middle Frontal Gyrus

$-30-657$

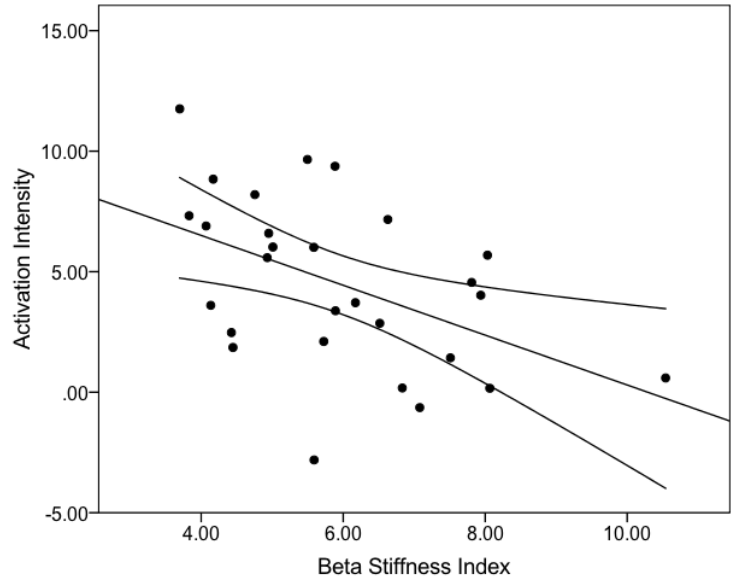

Fig. (2). Activation intensity versus BSI in the left precentral/middle frontal gyrus. 


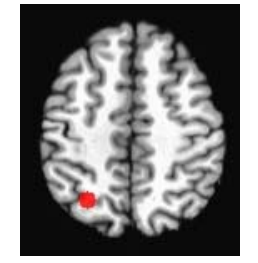

Left Superior Parietal Lobule/BA 7

$-26-6045$

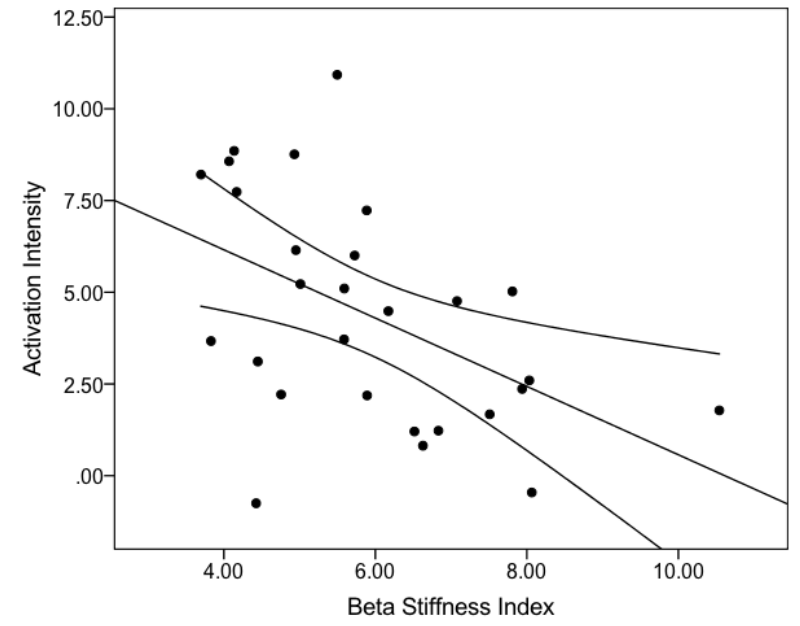

Fig. (3). Activation intensity versus BSI in the left superior parietal lobule/BA 7.

as between on 2-Back task blocks and task-related activation in the left precentral gyrus/BA $6(\mathrm{r}=0.40, \mathrm{p}=0.039)$ and in the left precentral $/$ middle frontal gyrus $(\mathrm{r}=0.41, \mathrm{p}=0.036)$.

\section{DISCUSSION}

The present study investigated the relationship between arterial stiffness and brain activation during a VWM task in a middle-aged sample with varying vascular risk. We chose a WM task based on previous research showing WM to be susceptible to vascular dysfunction [11]. We found significant negative correlations between $\beta$-stiffness index and task-related activation in the left precentral gyrus/BA 6, left precentral/middle frontal gyrus, and left superior parietal lobule/BA 7, as well as a strong trend between increased $\beta$ stiffness index and decreased task-related activation in the right superior frontal gyrus/BA 6. These relationships were independent of antihypertensive medication and hypertension status. Our results suggest that increased arterial stiffness is associated with subclinical alterations in the neural support of VWM systems.

Decreased task-related activation at comparable levels of performance is often interpreted as more efficient processing in a young healthy population. However, decreased taskrelated activation in our middle-aged population more likely reflects less efficient, an interpretation supported by previous research [11]. Indeed, an fMRI investigation of cortical activity during a VWM task in healthy adults varying in age found that while decreased activity corresponded to faster reaction time in the young group, increased activity corresponded to faster reaction time in the older group [28]. This study suggests that in middle-aged and older cohorts decreased brain activation during cognitive demand is likely associated with less efficient functioning. We found significant positive correlations between 0 -Back block accuracy and activation in the left precentral gyrus/BA 6 and the left superior parietal lobule/BA 7 and between 2-Back block accuracy and activation in the left precentral gyrus/BA 6 and left precentral/middle frontal gyrus. These findings support our interpretation that decreased activation in task-related areas reflects subclinical dysfunction in the cerebrovascular response to cognitive demands associated with VWM.
A prominent theory of VWM proposes a phonological loop consisting of a phonological short-term store and an articulatory subvocal rehearsal process [29]. The articulatory subvocal rehearsal process has been localized to areas in the frontal lobes, including the left precentral and left middle frontal gyri [30]. Presiding over the phonological loop is the central executive, which controls the processes of focusing, dividing, and switching attention [29]. One of the neural correlates proposed to subserve the central executive, specifically the control over focus of attention, is the superior parietal cortex/BA 7 [31]. Our finding of a negative correlation between $\beta$-stiffness index and activation in the left precentral gyrus/BA 6 , the left precentral/middle frontal gyrus, and the left superior parietal lobule/BA 7 suggests that greater arterial stiffness is associated with impaired functioning of the articulatory rehearsal and attentional focus components of VWM.

Several mechanisms could explain the relationship between increased arterial stiffness and decreased brain activation in response to cognitive demand. Arterial stiffness results from structural and functional changes in the arterial wall. Structural alterations include decreased elastin and increased collagen in the vessel wall, which reduce vessel wall elasticity [5]. In addition, arterial stiffness is associated with endothelial dysfunction, which impairs arterial reactivity [5]. It is logical that this reduced arterial compliance and reactivity characteristic associated with arterial stiffening may account for the reduced BOLD signal observed in our participants with greater BSI. The BOLD signal results from the delivery of oxygenated hemoglobin to brain areas with increased neuronal activity. In healthy individuals, this delivery of oxygenated blood exceeds neuronal demand. Our finding that increased $\beta$-stiffness index related to diminished BOLD signal but not to task performance suggests that increased arterial stiffness is associated with delivery of a reduced, but adequate, supply of oxygenated blood to activated brain regions.

In addition to dysfunctional cerebrovascular response to neuronal activity, arterial stiffness may contribute, over time, to cognitive decline by damaging cerebral microvasculature. Large artery elasticity serves to absorb pulsatile energy, 
thereby minimizing pulsatile load on microvasculature [32]. As elasticity decreases in large arteries, increased pulsatile energy is transmitted to the microcirculation, increasing the vulnerability of these vessels to pulse-induced damage [32]. Animal studies have demonstrated changes in cerebral microvascular structure and function resulting from increased pulse pressure [33]. This damage to the microvasculature may contribute to the pathogenesis of cerebral white matter lesions [34], which have been associated with decreased cognitive functioning [35] and dementia [36].

This is the first study to report a relationship between arterial stiffness and brain activation during performance of a cognitive task, indicating that BOLD fMRI may be a useful tool for identifying early markers of cognitive vulnerability in middle-aged, cognitively healthy individuals. Our results support previous research identifying arterial stiffness as a risk factor for cognitive decline and dementia [6-9]. A limitation of this study is the small sample size, which precluded examination of potential effects of ethnicity and sex. Similarly, the cross-sectional design of the study prevented us from assessing the relationship between arterial stiffness and brain functioning over time. Future studies should focus on arterial stiffness as an early marker of cognitive vulnerability and as a possible target for early intervention. Several studies focusing on the value of antihypertensive treatment for prevention of cognitive impairment have produced mixed results [37-40]. Although any antihypertensive agent that reduces mean arterial pressure can passively decrease arterial stiffness, not all of these treatments actively target arterial stiffness [41]. Further investigation of the effect of targeting arterial stiffness, either alone or with antihypertensive agents, on cognitive function and brain activation during cognitive demand may help identify more effective treatments of cognitive impairment associated with vascular risk. Finally, it is of note that there are a number of techniques available to measure arterial stiffness (e.g., pulse wave velocity, augmentation index, arterial compliance). Betastiffness index was chosen in the present study for several reasons. Beta-stiffness index is measured on the common carotid artery that leads to cerebral circulation downstream. As such, it is an ideal measure to assess relations between arterial stiffness and brain activation. Additionally, betastiffness index is much less affected by blood pressure [42] and can better tease out the relation between arterial stiffness and brain activation, both of which can be affected by blood pressure. However, future studies including multiple indexes of arterial stiffness in relation to brain function could be beneficial in determining which index may be most useful for studies of cognitive vulnerability.

\section{CONFLICT OF INTEREST}

The author(s) confirm that this article content has no conflicts of interest.

\section{ACKNOWLEDGEMENT}

This work was supported in part by the National Institute of Nursing Research Center Grant P30 NR005051, the National Institute of Neurological Disorders and Stroke R01 NS075565, the University of Texas Imaging Research Center, and Department of Psychology. The authors thank the UT Imaging Center Staff for their assistance with data collection.

\section{REFERENCES}

[1] Ishizaki T, Yoshida H, Suzuki T, et al. Effects of cognitive function on functional decline among community-dwelling nondisabled older Japanese. Arch Gerontol Geriatr 2006; 42(1): 47-58.

[2] Ferri CP, Prince M, Brayne C, et al. Global prevalence of dementia: a Delphi consensus study. Lancet 2005; 366(9503): 2112-2117.

[3] Breteler MM. Vascular risk factors for Alzheimer's disease: an epidemiologic perspective. Neurobiol Aging 2000; 21(2): 153-160.

[4] Safar ME, Levy BI, Struijker-Boudier H. Current perspectives on arterial stiffness and pulse pressure in hypertension and cardiovascular diseases. Circulation 2003; 107(22): 2864-2869.

[5] Zieman SJ, Melenovsky V, Kass DA. Mechanisms, pathophysiology, and therapy of arterial stiffness. Arterioscler Thromb Vasc Biol 2005; 25(5): 932-943.

[6] Fujiwara Y, Chaves PHM, Takahashi R, et al. Arterial pulse wave velocity as a marker of poor cognitive function in an elderly community-dwelling population. J Gerontol A Biol Sci Med Sci 2005; 60(5): 607-612.

[7] Hanon O, Haulon S, Lenoir H, et al. Relationship between arterial stiffness and cognitive function in elderly subjects with complaints of memory loss. Stroke 2005; 36(10): 2193-7.

[8] Jurasic M, Popovic IM, Morovic S, et al. Can beta stiffness index be proposed as risk factor for dementia. J Neurol Sci 2009; 283(12):13-16.

[9] Scuteri A, Tesauro M, Appolloni S, et al. Arterial stiffness as an independent predictor of longitudinal changes in cognitive function in the older individual. J Hypertens 2007; 25(5): 1035-1040.

[10] Haley AP, Sweet LH, Gunstad J, et al. Verbal working memory and atherosclerosis in patients with cardiovascular disease: an fMRI study. J Neuroimaging 2007; 17(3): 227-233.

[11] Jennings JR, Muldoon MF, Ryan C, et al. Reduced cerebral blood flow response and compensation among patients with untreated hypertension. Neurology 2005; 64(8): 1358-1365.

[12] Kannel WB. Blood pressure as a cardiovascular risk factor: Prevention and treatment. JAMA 1996; 275(20): 1571-1576.

[13] Kailasam MT, Parmer RJ, Tyrell EA, Henry RR, O'Connor DT. Circulating amylin in human essential hypertension: heritability and early increase in individuals at genetic risk. J Hypertens 2000; 18(11): 1611-1620.

[14] Page GD, France CR. Identifying hypertension using the Ohio Blood Pressure History Survey. Mil Med 2001; 166(3): 233-236.

[15] Chobanian AV, Bakris GL, Black HR, Cushman WC, Green LA, Izzo JL Jr, Jones DW, Materson BJ, Oparil S, Wright JT Jr, Roccella EJ. The Seventh Report of the Joint National Committee on Prevention, Detection, Evaluation, and Treatment of High Blood Pressure: the JNC 7 report. JAMA 2003; 289(19): 2560-72.

[16] Oldfield RC. The assessment and analysis of handedness: the Edinburgh inventory. Neuropsychologia 1971; 9(1):97-113.

[17] Braver TS, Cohen JD, Nystrom LE, et al. A parametric study of prefrontal cortex involvement in human working memory. Neuroimage 1997; 5(1): 49-62.

[18] Cortez-Cooper MY, Supak JA, Tanaka H. A new device for automatic measurements of arterial stiffness and ankle-brachial index. Am J Cardiol 2003; 91(12): 1519-1522, A9.

[19] Hirai T, Sasayama S, Kawasaki T, Yagi S. Stiffness of systemic arteries in patients with myocardial infarction. A noninvasive method to predict severity of coronary atherosclerosis. Circulation 1989; 80(1): 78-86.

[20] Lezak M. Neuropsychological Assessment. New York, NY: Oxford University Press; 1995.

[21] Folstein MF, Folstein SE, McHugh PR. "Mini-mental state". A practical method for grading the cognitive state of patients for the clinician. J Psychiatr Res 1975; 12(3): 189-198.

[22] Wechsler D. Wechsler Abbreviated Scale of Intelligence Manual. San Antonio: Harcourt Assessment Company; 1999.

[23] Beck A, Steer R, Brown G. BDI-II Manual. Fort Worth: The Psychological Corporation; 1996.

[24] Niezel M, Bernstein D, Russel R. Assessment of anxiety and fear. (Bellack A, Hersen M, eds.). Oxford: Pergamon Press; 1998.

[25] Cox RW. AFNI: software for analysis and visualization of functional magnetic resonance neuroimages. Comput Biomed Res 1996; 29(3): 162-173.

[26] Talairach J, Tournoux P. Co-planar stereotaxic atlas of the human brain 3-D proportional system: An approach to cerebral imaging. New York, NY: Thieme Medical Publishers; 1988. 
[27] Haley A, Gunstad J, Cohen R, et al. Neural correlates of visuospatial working memory in healthy young adults at risk for hypertension. Brain Imaging Behav 2008; 2(3): 192-199.

[28] Rypma B, D'Esposito M. Isolating the neural mechanisms of agerelated changes in human working memory. Nat Neurosci 2000; 3(5): 509-515.

[29] Baddeley A. Working memory: looking back and looking forward. Nat Rev Neurosci 2003; 4(10): 829-839.

[30] Smith EE, Jonides J. Working memory: a view from neuroimaging. Cogn Psychol 1997; 33(1): 5-42.

[31] Wager TD, Smith EE. Neuroimaging studies of working memory: a meta-analysis. Cogn Affect Behav Neurosci 2003; 3(4): 255-274.

[32] Mitchell GF, Parise H, Benjamin EJ, et al. Changes in arterial stiffness and wave reflection with advancing age in healthy men and women: The Framingham Heart Study. Hypertension 2004; 43(6): 1239-1245.

[33] Baumbach GL. Effects of increased pulse pressure on cerebral arterioles. Hypertension 1996; 27(2): 159-167.

[34] Ohmine T, Miwa Y, Yao H, et al. Association between arterial stiffness and cerebral white matter lesions in community-dwelling elderly subjects. Hypertens Res 2008; 31(1): 75-81.

[35] Au R, Massaro JM, Wolf PA, et al. Association of white matter hyperintensity volume with decreased cognitive functioning: The Framingham Heart Study. Arch Neurol 2006; 63(2): 246-250.
[36] Prins ND, van Dijk EJ, den Heijer T, et al. Cerebral white matter lesions and the risk of dementia. Arch Neurol 2004; 61(10): 15311534.

[37] Knecht S, Wersching H, Lohmann H, et al. High-normal blood pressure is associated with poor cognitive performance. Hypertension 2008; 51(3): 663-668.

[38] Prince MJ, Bird AS, Blizard RA, Mann AH. Is the cognitive function of older patients affected by antihypertensive treatment? Results from 54 months of the Medical Research Council's trial of hypertension in older adults. BMJ 1996; 312(7034): 801-805.

[39] Birns J, Morris R, Donaldson N, Kalra L. The effects of blood pressure reduction on cognitive function: a review of effects based on pooled data from clinical trials. J Hypertens 2006; 24(10):19071914.

[40] Saxby BK, Harrington F, Wesnes KA, McKeith IG, Ford GA. Candesartan and cognitive decline in older patients with hypertension: a substudy of the SCOPE trial. Neurology 2008; 70:18581866.

[41] Van Bortel LM, Struijker-Boudier HA, Safar ME. Pulse pressure, arterial stiffness, and drug treatment of hypertension. Hypertension 2001; 38(4): 914-921.

[42] Hirai T, Sasayama S, Kawasaki T, Yagi S. Stiffness of systemic arteries in patients with myocardial infarction: A noninvasive method to predict severity of coronary atherosclerosis. Circulation 1989; 80: 78-86.

Received: November 11, 2013

Revised: January 02, 2014

Accepted: January 10, 2014

(C) Steward et al.; Licensee Bentham Open.

This is an open access article licensed under the terms of the Creative Commons Attribution Non-Commercial License (http://creativecommons.org/licenses/by-nc/3.0/) which permits unrestricted, non-commercial use, distribution and reproduction in any medium, provided the work is properly cited. 